\title{
Comparing Medical Utilization and Cost Outcomes in Oral Versus Injectable Immunotherapy Users with Chronic Inflammatory Joint and Skin Diseases
}

\author{
Rituparna Bhattacharya, PhD; Katie Herren, PharmD, MS; Insiya Poonawalla, PhD; \\ Suvapun Bunniran, PhD; Andrea Bloomfield, PharmD, BCPS; and Phil Schwab, PhD
}

\begin{abstract}
BACKGROUND: Rheumatoid arthritis (RA), psoriatic arthritis (PsA), and psoriasis (PSO) are immune-mediated systemic, chronic inflammatory conditions. Moderate to severe disease is treated with conventional disease-modifying antirheumatic drugs (DMARDs) such as methotrexate, sulfasalazine, or leflunomide. If a patient does not respond to these firstline treatments, then tumor necrosis factor inhibitor (TNFi) or non-TNFi immunotherapy agents are administered via infusion, injection, or taken orally. Although the effectiveness of established infusion, injection, and newer oral therapies are known, the relative effectiveness among the routes of administration is not well understood.
\end{abstract}

OBJECTIVE: To compare drug use, health care resource utilization, and costs among patients who are treatment-naive to oral immunotherapy and injectable biologic immunotherapy.

METHODS: This retrospective observational study used claims data from a large U.S. health plan to identify new users of oral and injectable immunotherapy, diagnosed with a joint (RA or PsA), skin (PSO), or joint and skin condition from July 1, 2014, to June 30, 2017. The index date was the first claim for an oral or injectable medication. Medicaid, Medicare Advantage, and commercial plan patients aged 19-89 years with continuous enrollment 6 months before and 12 months after the index date were included in the study. Outcomes were adjusted using propensity score by inverse probability of treatment weighting. Treatment discontinuation, switching, health care resource utilization, and costs were measured during the post-index period.

RESULTS: Oral versus injectable users with joint ( $n=458$ vs. 3,875$)$, skin ( $n=265$ vs. 951$)$, or joint and skin ( $n=171$ vs. 805$)$ conditions were identified. For drug utilization outcomes, no differences in discontinuation rates were observed between oral and injectable groups for any of the cohorts. However, those in skin and joint and skin cohorts had higher rates of switching to other immunotherapies in patients initiated on orals compared with injectables. Health care resource utilization outcomes were mixed. While mean outpatient and physician office visits were significantly higher in oral compared with injectable groups across all 3 cohorts, no differences were observed for inpatient stays. Total costs (medical plus pharmacy) were lower for oral groups across all 3 cohorts. Pharmacy costs were lower for oral groups, but medical costs were higher for oral groups across all 3 cohorts.

CONCLUSIONS: This is the first population-level study at a route-ofadministration level, which compared switching, health care resource utilization, and costs across several conditions. Switching drugs was more likely in the oral group, which may indicate lower effectiveness or tolerability of oral immunotherapies relative to injectables. Health care resource utilization was higher in the oral group, but total costs were lower, which was likely driven by the lower costs of oral drugs.

J Manag Care Spec Pharm. 2020;26(10):1246-56

Copyright $\odot 2020$, Academy of Managed Care Pharmacy. All rights reserved.

\section{What is already known about this subject}

Efficacy of injectable and oral immunotherapies for the treatment of moderate to severe rheumatoid arthritis, psoriatic arthritis, and psoriasis is well established.

Injectable immunotherapies have been available longer than oral options, so real-world evidence is more widely available for injectables.

Discontinuation of immunotherapy or switching to another treatment is frequent and could be a result of lack of effectiveness, cost burden, or side effects for patients using injectable or oral therapies or a result of injection-related discomfort for patients using injectable therapies.

\section{What this study adds}

This study included real-world evidence for evaluating injectable and oral immunotherapies based on treatment patterns, health care resource utilization, and cost.

Patients using oral therapies were more likely to switch to another treatment than users of injectables, possibly indicating lower tolerability and/or effectiveness of oral therapies.

Patients using oral therapies had lower total costs driven by lower costs of oral therapies than injectable drugs.

$\mathrm{R}$ heumatoid arthritis (RA), psoriatic arthritis (PsA), and psoriasis (PSO) are immune-mediated systemic and chronic inflammatory conditions of the joint and skin that affect approximately $0.2 \%-3.1 \%$ of the U.S. population. ${ }^{1-3}$ These diseases have different pathologies with persistent pain and chronic inflammation resulting from structural modifications of the bone and cartilage. ${ }^{4}$ Once moderately or severely progressed, the conditions can have a significant, negative effect on a patient's ability to perform activities of daily living and on health-related quality of life.,5 Therapeutic management for these conditions has evolved, and treatment recommendations are based on disease duration and/or severity.

Patients with early symptomatic RA, or low RA disease activity, are typically treated with traditional disease-modifying antirheumatic drugs (DMARDs), such as methotrexate, hydroxychloroquine, leflunomide, or sulfasalazine. However, 
as the condition progresses to moderate or high disease activity, patients may receive therapy with tumor necrosis factor inhibitor (TNFi) biologics (e.g., adalimumab, certolizumab pegol, etanercept, golimumab, and infliximab) or non-TNFi biologics (e.g., abatacept, rituximab, and tocilizumab). ${ }^{7}$ Similarly, patients with PsA are initially treated with nonsteroidal antiinflammatory agents or intra-articular corticosteroid injections for mild disease or traditional DMARDs for moderate disease and transition to TNFi or non-TNFi biologic (e.g., secukinumab) therapy as the condition progresses or responds inadequately to previous treatment. ${ }^{8} \mathrm{PSO}$, on the other hand, is treated with topical agents for limited disease and ultraviolet light therapy (e.g., narrowband and broadband UVB, UVA); systemic therapy (e.g., acitretin); or biologic agents (e.g., adalimumab, etanercept, infliximab, certolizumab, ustekinumab, secukinumab, ixekizumab, brodalumab, guselkumab, tildrakizumab, and risankizumab) for extensive disease. ${ }^{9,10}$ Patients with PSO who also have PsA may receive upfront treatment with a TNFi with or without methotrexate. ${ }^{8}$

Although treatments for these chronic conditions are effective, discontinuation of medication is a known problem. About $17 \%$ of patients with RA and $27 \%$ with PsA reported discontinuation of their TNFi or anti-interleukin inhibitor biologic therapies within the 12 months of follow-up. ${ }^{11,12}$ Similarly, $27 \%-37 \%$ of patients with moderate to severe PSO discontinued treatment with biologics in the first year compared with 18\% of patients with mild disease. ${ }^{13}$ Treatment discontinuation may be due to lack of effectiveness, side effects, or cost burden. In addition, the cyclical dosing with intravenous and subcutaneous routes of administration often leads to unpleasant physical experiences (e.g., pain, discomfort, burning, redness, swelling, and tolerability) or psychological perceptions (e.g., anxiety, fear of injections, and long-term safety), ${ }^{14-16}$ making alternative routes of administration (e.g., oral) extremely appealing for many patients over the established injectable and infusible treatment options.

In the last few years, 2 oral agents became available as alternative treatments to injectable routes of administration: Tofacitinib, a Janus kinase inhibitor, was approved for patients with moderate to severe RA in $2012,{ }^{17}$ while apremilast, a small molecule phosphodiesterase-4-inhibitor, was approved for use in patients with PsA and PSO in $2014 .{ }^{18}$ A series of phase 3 clinical trials have consistently shown reduced RA symptoms and improved patient-reported outcomes with manageable safety profiles in the treatment arm receiving oral tofacitinib. The ORAL Strategy trial, which compares tofacitinib monotherapy, tofacitinib plus methotrexate, and injectable adalimumab plus methotrexate in patients with a previous inadequate response to methotrexate, reported an American College of Rheumatology (ACR) 50 score in 38\%, $46 \%$, and $44 \%$ of patients at 6 months follow-up, respectively, indicating that tofacitinib plus methotrexate was noninferior to the injectable adalimumab plus methotrexate combinations. ${ }^{19}$ The PALACE phase 3 clinical trial programs of apremilast reported reduced symptoms of PsA, as well as improved physical functioning, for patients in the treatment arm compared with placebo, ${ }^{20-23}$ while the ESTEEM phase 3 clinical trial programs demonstrated a reduction in the severity of moderate to severe plaque psoriasis, as well as improvement in quality of life among patients receiving oral apremilast therapy compared with placebo. ${ }^{24,25}$

Although the efficacy of tofacitinib and apremilast has been demonstrated, data on effectiveness in real-world settings are minimal. ${ }^{26-28}$ With easy administration and adherence/persistence akin to biologic immunotherapies, ${ }^{26,27,29}$ oral therapies are receiving greater acceptance in clinical practice. However, there is a scarcity of evidence on how effectiveness of oral agents compares with injectable immunotherapies on various health care utilization and cost outcomes across different chronic inflammatory diseases. The objective of this study was to compare treatment discontinuation, treatment switching, health care utilization, and costs between patients receiving injectable immunotherapies versus oral therapies among those diagnosed with RA, PsA, and PSO.

\section{Methods}

\section{Data Source, Study Design, and Sample Selection}

This was a retrospective cohort study using health care administrative claims data. The study sample was selected from the Humana Research Database (Louisville, KY). Humana is a health and well-being company serving millions of people across the United States through Medicare Advantage Prescription Drug (MAPD) plans, stand-alone Prescription Drug Plans (PDPs), Medicaid, and commercial plan offerings. The Humana Research Database has national coverage with a high proportion of individuals from Texas, Florida, and Ohio. For this study, medical claims and pharmacy claims for the MAPD, Medicaid, and commercial populations were examined. Claims data included information regarding physician visits, outpatient visits, emergency department (ED) visits, and inpatient hospitalizations. Pharmacy claims data included detailed information for each prescription fill. The full study period was from July 1, 2014, to June 30, 2017.

The study protocol was approved by an independent institutional review board (Schulman Associates IRB, now Advarra IRB, \#201707055).

\section{Inclusion and Exclusion Criteria}

The study sample included patients aged 19-89 years with at least 1 pharmacy or medical claim for an oral or injectable immunotherapy agent from January 1, 2015, to June 30, 2016. The earliest claim was set as the index date. Claims for oral immunotherapies tofacitinib and apremilast were identified first to establish the oral treatment population. 


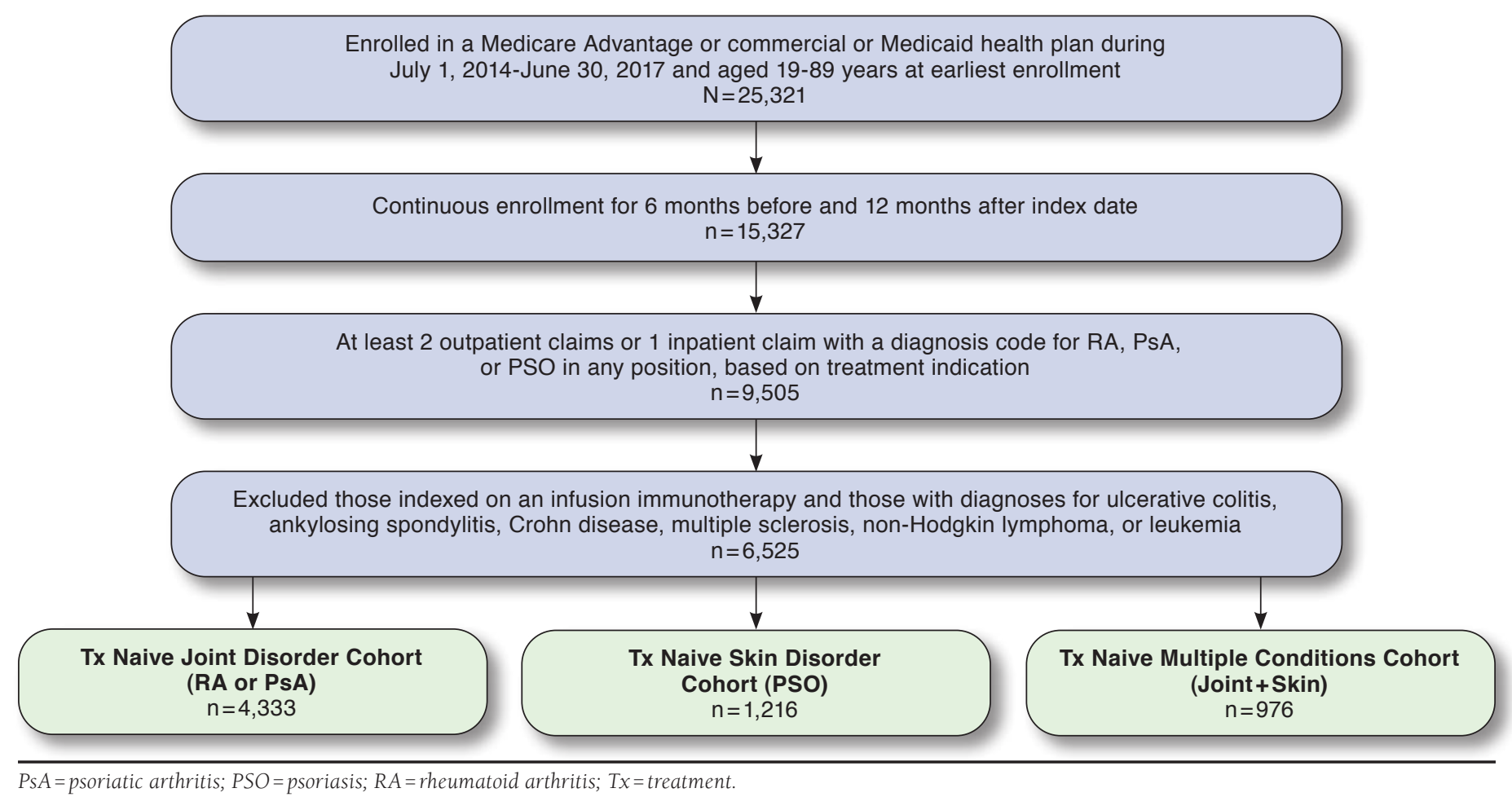

Among patients without an oral immunotherapy, injectable claims for the following products were identified: adalimumab, etanercept, abatacept, certolizumab pegol, golimumab, tocilizumab, anakinra, ustekinumab, secukinumab, ixekizumab, or sarilumab. Generic Product Identifier (GPI) codes and Common Procedural Terminology (CPT)/Healthcare Common Procedure Coding System (HCPCS) codes Supplementary Table 1, available in online article) were used to identify immunotherapies. A 6-month washout period before the index date was established so that patients were naive to any of the aforementioned oral or injectable immunotherapies and naive to infusible immunotherapies (infliximab, abatacept, tocilizumab, natalizumab, golimumab, ustekinumab, rituximab, and vedolizumab).

Patients were also required to have at least 1 inpatient or 2 outpatient diagnosis codes for RA (International Classification of Diseases, Ninth/Tenth Revision, Clinical Modification [ICD9-CM] codes 714.0, 714.1, 714.2, 714.4, 714.81; ICD-10-CM codes M05, M06.0, and M06.2-M06.9); PsA (ICD-9-CM code 696.0; ICD-10-CM code L40.5); or PSO (ICD-9-CM code 696.1; ICD-10-CM codes L40.0-L40.4, L40.8, and L40.9) during the study period, with continuous enrollment for 6 months before and 12 months after the index date.
Patients with ankylosing spondylitis, hidradenitis suppurativa, uvetitis or juvenile idiopathic arthritis were excluded from the study. Further, patients with a claim for natalizumab and diagnosis for multiple sclerosis were excluded, as were patients with a claim for rituximab and diagnosis for non-Hodgkins lymphoma or chronic lymphocytic leukemia during the study period.

Patients meeting the study eligibility criteria were classified into 3 condition-specific cohorts: (1) joint (i.e., RA or PsA), (2) skin (i.e., PSO), and (3) joint and skin (i.e., an indication for both joint and skin conditions was required).

\section{Baseline Measures}

Demographic characteristics measured at baseline included age; sex; health plan type (health maintenance organization, preferred provider organization, point of service, fee for service, or other); geographic region (Northeast, Midwest, South, and West) ${ }^{30}$; and population density groups (rural, suburban, urban, or unknown). ${ }^{31,32}$

Measures of baseline health status or severity included the Deyo-Charlson Comorbidity Index (DCI) and RxRisk-V scores. ${ }^{33-36}$ The DCI score is based on 17 categories of comorbidities (Supplementary Table 2, available in online article) to 


\section{Comparing Medical Utilization and Cost Outcomes in Oral Versus Injectable Immunotherapy Users with Chronic Inflammatory Joint and Skin Diseases}

TABLE 1 Demographic Characteristics of Treatment-Naive Patients

\begin{tabular}{|c|c|c|c|c|c|c|}
\hline \multirow[b]{2}{*}{ Characteristics } & \multicolumn{3}{|c|}{ Before IPTW } & \multicolumn{3}{|c|}{ After IPTW } \\
\hline & Oral & Injectable & SMD & Oral & Injectable & SMD \\
\hline \multirow[t]{2}{*}{ Joint only } & $\mathrm{n}=458$ & $\mathrm{n}=3,875$ & & & & \\
\hline & Mean & Mean & & Weighted Mean & Weighted Mean & \\
\hline Age & 59.4 & 59.1 & 0.021 & 58.8 & 59.2 & -0.032 \\
\hline DCI score ${ }^{a}$ & 1.6 & 1.4 & 0.178 & 1.4 & 1.4 & 0.019 \\
\hline RxRisk score ${ }^{a}$ & 6.9 & 6.4 & 0.158 & 6.4 & 6.4 & 0.004 \\
\hline \multirow[t]{2}{*}{ Log of pre-index total costs ${ }^{\mathrm{a}}$} & $\$ 17,619$ & $\$ 18,152$ & -0.121 & $\$ 18,813$ & $\$ 18,073$ & -0.003 \\
\hline & $\%$ & $\%$ & & Weighted \% & Weighted \% & \\
\hline Pre-index inpatient stay ${ }^{a}$ & 10.0 & 7.3 & 0.099 & 8.0 & 7.6 & 0.014 \\
\hline Pre-index ED visita & 27.3 & 21.2 & 0.143 & 22.7 & 21.9 & 0.019 \\
\hline Sex $=$ female & 77.7 & 77.3 & 0.034 & 78.2 & 77.5 & 0.016 \\
\hline \multicolumn{7}{|l|}{ Region } \\
\hline South & 76.6 & 74.8 & 0.043 & 75.1 & 75.0 & 0.001 \\
\hline NE/MW/West & 23.4 & 25.2 & & 24.9 & 25.0 & \\
\hline \multicolumn{7}{|l|}{ Population density } \\
\hline Urban & 64.0 & 64.3 & -0.007 & 63.4 & 64.3 & -0.018 \\
\hline Not urban & 36.0 & 35.7 & & 36.6 & 35.7 & \\
\hline \multicolumn{7}{|c|}{ Program in which patient is enrolled } \\
\hline MAPD & 71.8 & 64.6 & 0.157 & 65.1 & 65.6 & -0.012 \\
\hline Commercial/Medicaid & 28.2 & 35.4 & & 34.9 & 34.4 & \\
\hline \multicolumn{7}{|l|}{ Plan typeb } \\
\hline $\mathrm{HMO}$ & 47.2 & 49.1 & -0.039 & 49.2 & 48.8 & 0.008 \\
\hline Non-HMO & 52.8 & 50.9 & & 50.8 & 51.2 & \\
\hline \multirow[t]{2}{*}{ Skin only } & $\mathrm{n}=265$ & $\mathrm{n}=951$ & & & & \\
\hline & Mean & Mean & & Weighted Mean & Weighted Mean & \\
\hline Age & 56.7 & 52.9 & 0.279 & 52.9 & 53.8 & -0.055 \\
\hline DCI score & 0.65 & 0.46 & 0.279 & 0.54 & 0.50 & -0.055 \\
\hline RxRisk score ${ }^{a}$ & 5.15 & 4.59 & 0.176 & 4.78 & 4.76 & 0.006 \\
\hline \multirow[t]{2}{*}{ Pre-index total costs ${ }^{\mathrm{a}}$} & $\$ 11,122$ & $\$ 15,833$ & -0.453 & $\$ 16,638$ & $\$ 14,994$ & 0.033 \\
\hline & $\%$ & $\%$ & & Weighted \% & Weighted \% & \\
\hline Pre-index inpatient staya & 5.3 & 4.5 & 0.035 & 6.5 & 4.8 & 0.071 \\
\hline Pre-index ED visita & 20.4 & 14.0 & 0.170 & 15.7 & 15.6 & 0.003 \\
\hline Sex =female & 53.2 & 47.1 & 0.154 & 46.4 & 48.7 & 0.045 \\
\hline \multicolumn{7}{|l|}{ Region } \\
\hline South & 74.3 & 78.2 & -0.092 & 79.6 & 77.5 & 0.05 \\
\hline NE/MW/West & 25.7 & 21.8 & & 20.4 & 22.5 & \\
\hline \multicolumn{7}{|l|}{ Population density } \\
\hline Urban & 70.6 & 70.7 & -0.065 & 69.2 & 70.4 & 0.030 \\
\hline Not urban & 29.4 & 29.3 & & 30.8 & 29.6 & \\
\hline \multicolumn{7}{|c|}{ Program in which patient is enrolled } \\
\hline MAPD & 50.6 & 38.6 & 0.243 & 38.8 & 41.5 & -0.054 \\
\hline Commercial/Medicaid & 49.4 & 61.4 & & 61.2 & 58.5 & \\
\hline \multicolumn{7}{|l|}{ Plan type } \\
\hline $\mathrm{HMO}$ & 52.8 & 56.0 & -0.065 & 56.5 & 55.1 & 0.030 \\
\hline Non-HMO & 47.2 & 44.0 & & 43.5 & 44.9 & \\
\hline
\end{tabular}

calculate a score that reflects the cumulative increased likelihood of 1-year mortality. ${ }^{37}$ The evolution of the Deyo-Charlson methodology since its origin has permitted researchers to use the score as an assessment of overall patient health risk, typically performed at baseline in a given study. It uses diagnosis and procedure codes to identify the presence of each category, ${ }^{34,37}$ which has a weight of 1-6 (the composite score can range from 0-33). 


\section{Comparing Medical Utilization and Cost Outcomes in Oral Versus Injectable Immunotherapy Users with Chronic Inflammatory Joint and Skin Diseases}

TABLE 1 Demographic Characteristics of Treatment-Naive Patients (continued)

\begin{tabular}{|c|c|c|c|c|c|c|}
\hline \multirow[b]{2}{*}{ Characteristics } & \multicolumn{3}{|c|}{ Before IPTW } & \multicolumn{3}{|c|}{ After IPTW } \\
\hline & Oral & Injectable & SMD & Oral & Injectable & SMD \\
\hline \multirow[t]{2}{*}{ Joint and skin conditions } & $\mathrm{n}=171$ & $\mathrm{n}=805$ & & & & \\
\hline & Mean & Mean & & Weighted Mean & Weighted Mean & \\
\hline Age & 57.4 & 55.5 & 0.143 & 55.2 & 55.8 & -0.051 \\
\hline DCI score ${ }^{a}$ & 0.88 & 0.82 & 0.041 & 0.82 & 0.82 & 0.002 \\
\hline RxRisk score ${ }^{a}$ & 6.20 & 5.76 & 0.137 & 5.98 & 5.85 & 0.039 \\
\hline \multirow[t]{2}{*}{ Pre-index total costs ${ }^{a}$} & $\$ 15,564$ & $\$ 17,778$ & -0.216 & $\$ 18,024$ & $\$ 17,357$ & 0.004 \\
\hline & $\%$ & $\%$ & & Weighted \% & Weighted \% & \\
\hline Pre-index inpatient stay ${ }^{a}$ & 5.8 & 6.6 & -0.031 & 7.0 & 6.5 & 0.02 \\
\hline Pre-index ED visit ${ }^{\mathrm{a}}$ & 17.0 & 19.6 & -0.069 & 21.1 & 19.2 & 0.046 \\
\hline Sex =female & 64.9 & 57.5 & 0.159 & 60.9 & 59.0 & 0.059 \\
\hline \multicolumn{7}{|l|}{ Region } \\
\hline South & 75.4 & 76.9 & -0.034 & 75.3 & 76.6 & -0.03 \\
\hline NE/MW/West & 24.6 & 23.1 & & 24.7 & 23.4 & \\
\hline \multicolumn{7}{|l|}{ Population density } \\
\hline Urban & 66.1 & 66.1 & -0.000 & 65.9 & 66.1 & -0.005 \\
\hline Not urban & 33.9 & 33.9 & & 34.1 & 33.9 & \\
\hline \multicolumn{7}{|c|}{ Program in which patient is enrolled } \\
\hline MAPD & 58.5 & 53.8 & 0.095 & 54.3 & 54.8 & -0.009 \\
\hline Commercial/Medicaid & 41.5 & 46.2 & & 45.7 & 45.2 & \\
\hline \multicolumn{7}{|l|}{ Plan type } \\
\hline $\mathrm{HMO}$ & 52.6 & 54.7 & -0.041 & 51.8 & 54.2 & -0.047 \\
\hline Non-HMO & 47.4 & 45.3 & & 48.2 & 45.8 & \\
\hline
\end{tabular}

a Measured during 6-month pre-index period.

${ }^{b}$ Non-HMO plans include fee for service, preferred provider organization, point of service, and other plan types

$D C I=$ Deyo-Charlson Comorbidity Index; $E D=$ emergency department; HMO = health maintenance organization; IPTW =inverse proportion of treatment weight; $M A P D=$ Medicare Advantage with Prescription Drug plan; $M W=$ Midwest $N E=$ Northeast R $x$ Risk $=R \times R i s k-V$ index; SMD = standardized mean difference.

On the other hand, the RxRisk-V is a pharmacy-based comorbidity index originally developed as an enhancement of the RxRisk risk assessment instrument for use in the Veterans Health Administration population. ${ }^{36}$ The RxRisk-V is based on the identification of 45 distinct medical condition categories via their associated medication treatments (Supplementary Table 3, available in online article). The RxRisk-V score is determined by summing the number of unique condition categories, such that a higher score indicates a greater comorbidity burden.

DCI and RxRisk-V scores were calculated using claims from the 6-month pre-index period. Physician specialty was identified for the index immunotherapy claim and defined based on National Provider Identifier codes and the Healthcare Provider Taxonomy Code Set. ${ }^{38}$ Pre-index health care resource utilization included medical claims for inpatient and ED visits in the 6 months before the index date.

\section{Outcome Measures}

Treatment discontinuation was measured as a gap that exceeded 45 days between the end of the days supply of the previous prescription and the next prescription fill for the index oral or injectable immunotherapy or before switching to another agent. ${ }^{39}$ Aligning with previous studies, this gap was chosen to accommodate the dosing intervals for the various immunotherapies and allow an additional buffer period. . $9,39-41^{2}$ Immunotherapy drug switch was measured for the first switch and defined as the first pharmacy or medical claim for a different immunotherapy agent within 45 days after the last days supply of the index immunotherapy through the end of the12-month post-index period.

Health care resource utilization was assessed in the 12-month post-index period and identified based on place of service. Inpatient episodes of care were derived from facility claims and identified by bill type and revenue codes, as well as dates of service. ED visits were identified using revenue codes, place of treatment codes, and CPT and HCPCS codes. Physician office visits were classified by CPT/HCPCS and Berenson-Eggers Type of Service categorization, while outpatient visits were calculated using the place of treatment code.

All-cause, medical only, and immunotherapy-related medical and pharmacy costs were determined for the 12-month post-index period and included patient and health plan-paid costs. Costs for all services were adjusted to 2016 U.S. dollars using the medical component of the Consumer Price Index. 


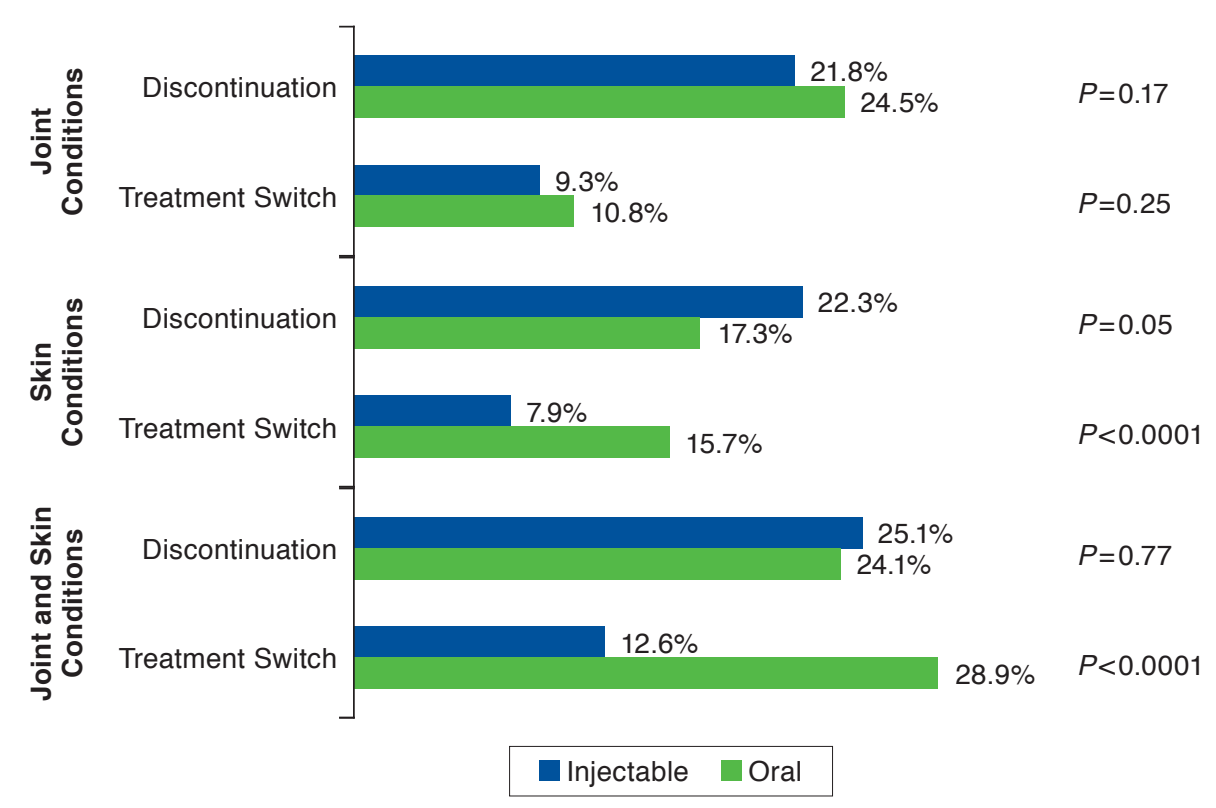

Note: Percentages represent IPTW-adjusted percentages. P values were obtained from chi-square tests.

IPTW=inverse probability of treatment weight.

All-cause costs included all medical and pharmacy claims during the period. Medical costs included all medical costs summed across all medical claims that were not related to any immunotherapy drug claim as identified by HCPCS codes. Immunotherapy-related costs included costs summed across all medical and pharmacy claims for oral and injectable immunotherapies that were the focus of the study.

\section{Statistical Analysis Plan}

Given nonrandom treatment assignment in routine clinical care, a propensity score-based method known as inverse probability of treatment weighting (IPTW) was used to mitigate selection bias arising from baseline differences in the patient and clinical characteristics between the injectable and oral treatment groups. A logistic regression model was used to estimate a propensity score (e.g., conditional probability of being treated given the observed baseline covariates) for each patient, which was applied to the IPTW technique. With IPTW, each individual was assigned a weight calculated as the inverse probability (p) of receiving the treatment they actual received. Hence, patients in the oral immunotherapy group received a weight of $1 / p$, while patients in the injectable immunotherapy group received a weight of $1 /(1-p)$.
Logistic regression models used to calculate the propensity scores controlled for baseline demographic characteristics, measures of health status or severity, pre-index health care resource use, and provider specialty. The weights were included in the appropriate outcome models to balance the distribution of potential confounders across groups. ${ }^{42}$ Frequency and percentages weighted by inverse probability weights were reported for treatment groups, and chi-square tests were conducted to assess the statistical significance of the association between the treatment groups and the probability of treatment discontinuation, switching, inpatient hospitalizations, and ED admissions. Means with standard errors (SE) adjusted for inverse probability were reported for continuous variables, such as total number of physician office and outpatient visits and cost estimates.

Generalized linear models (GLM) with Poisson distribution and log link functions were fit to evaluate the total number of physician office and outpatient visits. GLMs with gamma variance and log link functions were used to assess the statistical significance of the association between treatment groups and various cost metrics. GLM models controlled for only the index drug type. All comparisons were reported separately for each condition type: joint, skin, and joint and skin. All statistical analyses were conducted using SAS Enterprise Guide 7.1 (SAS Institute, Cary, NC). 


\section{FIGURE 3 IPTW-Adjusted Comparison of Health Care Resource Utilization Outcomes Observed During 1-Year} Follow-up of Oral vs. Injectable Users

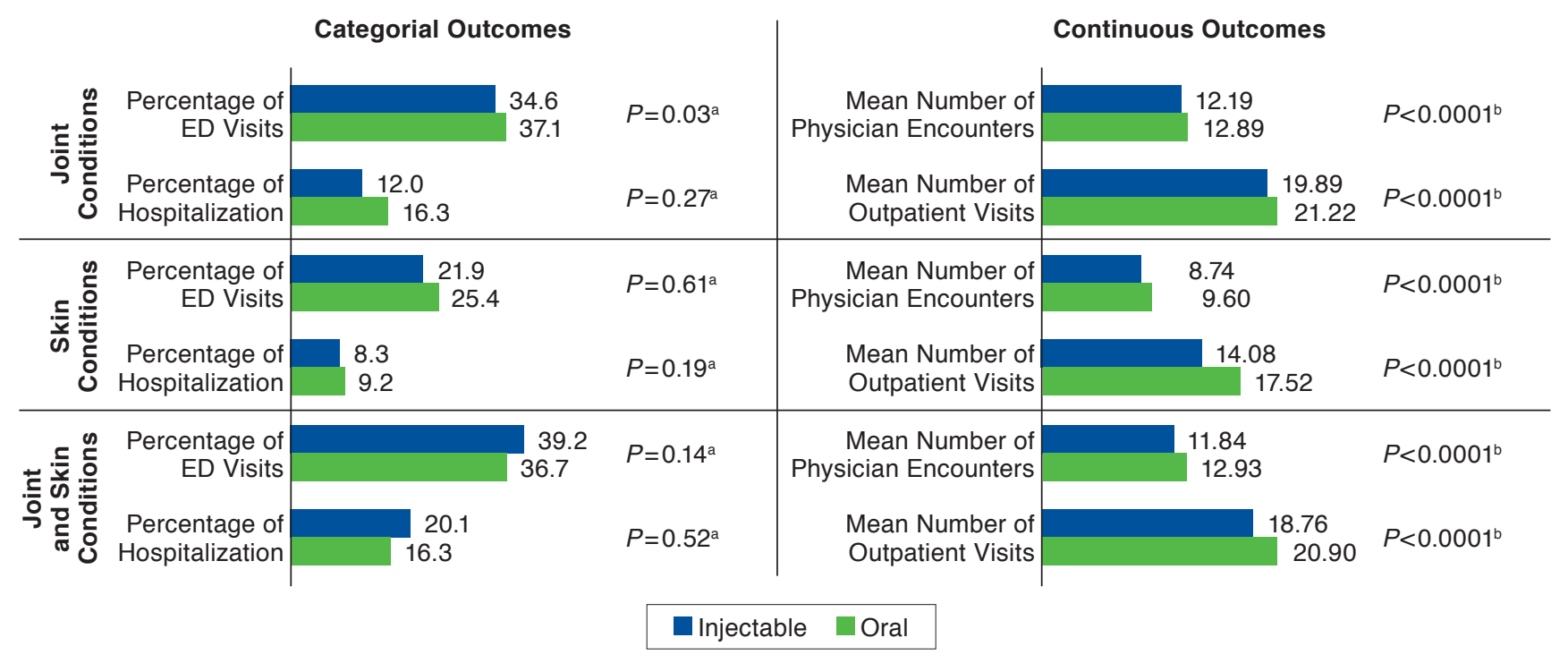

aP values were obtained from chi-square tests.

${ }^{b} P$ values were obtained from IPTW-adjusted generalized linear model that controlled only for index drug.

$E D=$ emergency department; IPTW=inverse probability of treatment weight.

\section{Results}

Of the 6,525 patients meeting the study eligibility criteria (Figure 1), 66\% ( $\mathrm{n}=4,333)$ were classified with joint conditions, 19\% ( $\mathrm{n}=1,216)$ with skin conditions, and $15 \%(\mathrm{n}=976)$ with joint and skin conditions. A majority of patients in each study cohort were indexed to an injectable agent: 89\% $(n=3,875)$ for joint, 78\% $(n=951)$ for skin, and 82\% $(n=805)$ for joint and skin. After applying weights, standardized mean differences were relatively small. There were no significant differences between the oral and injectable groups for joint, skin, or joint and skin conditions based on measures of baseline demographic characteristics and health status (Table 1).

No differences in discontinuation rates were observed between oral and injectable groups for any of the cohorts. However, weighted comparisons found higher occurrence of switching to another immunotherapy for patients initiated on orals compared with injectables in the skin (oral vs. injectable: $15.7 \%$ vs. $7.9 \%$; chi-square $P<0.0001$ ) and joint and skin (oral vs. injectable: $28.9 \%$ vs. $12.6 \%$; chi-square $P<0.0001$ ) cohorts (Figure 2).

Health care utilization results in the 12-month follow-up period were mixed. Greater mean outpatient visits were observed for patients indexing to an oral immunotherapy in the joint cohort, skin cohort, and joint and skin cohort. Similarly, greater mean physician encounters were observed for patients indexing to oral immunotherapy in the joint cohort, skin cohort, and joint and skin cohorts. More patients using an oral therapy had an ED visit in the joint cohort (oral vs. injectable: $37.1 \%$ vs. $34.6 \%$; chi-square $P<0.03$ ), but differences were not observed for the skin or joint and skin cohorts (Figure 3 and Supplemental Table 4, available in online article).

Although medical costs were lower for the injectable group compared with the oral group for all 3 study cohorts, pharmacy costs and medical and pharmacy costs were higher for the injectable group compared with the oral group (Figure 4 and Supplemental Table 4, available in online article).

\section{Discussion}

To our knowledge, this is the first population-level study at a route-of-administration level to compare injectable and oral biologic immunotherapies on drug utilization and medical utilization outcomes, as well as costs of care, across various immune-mediated systemic inflammatory diseases. This study found that injectable users were less likely than oral users to switch medications, despite a similar likelihood of treatment discontinuation between the groups. Few real-world studies have compared treatment patterns separately for oral tofacitinib or apremilast compared with biologic therapies. In a study by Machado et al. (2018), results similar to this study were observed, wherein fewer patients with RA on TNFi or non-TNFi biologics switched medications relative to patients on oral tofacitinib. ${ }^{28}$ In contrast, studies by Wu et al. (2019) 
FIGURE 4 IPTW-Adjusted Mean Health Care Outcomes Observed During 1-Year Follow-up of Oral vs. Injectable Users

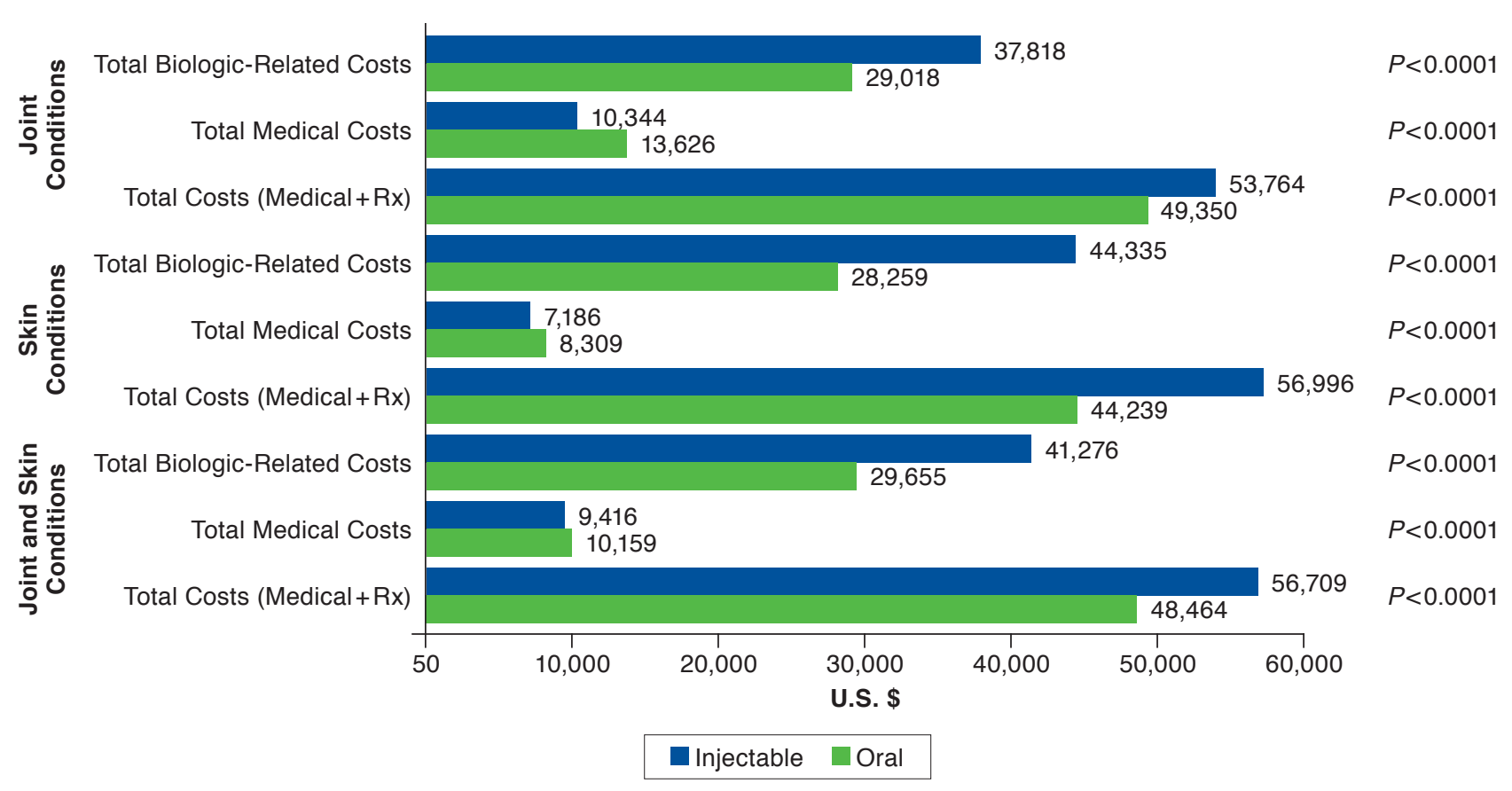

Note: P values were obtained from IPTW-adjusted generalized linear model that controlled only for index drug. IPTW=inverse probability of treatment weight; $R x=$ prescription.

showed that switching rates were similar among patients initiating oral apremilast versus biologics in those with PSO or PsA. ${ }^{26,27}$ Our finding of greater switching within skin and joint and skin cohorts indicates that oral immunotherapy in general might not be as effective and/or tolerated as injectable immunotherapies in the treatment of these chronic conditions because switching medications is considered a potential proxy for lack of treatment effect. ${ }^{43}$ Switching was also marginally greater for the joint cohort, although lacked statistical significance.

Aligning with the results around switching, injectable users also had fewer physician encounters and fewer outpatient visits than oral users consistently across all condition cohorts. If adverse effects and treatment intolerance are drivers for switching, it is not surprising to observe higher physician visits and outpatient encounters for patients on oral therapy, particularly tofacitinib. While all of the immunological treatments included in this study function to suppress the immune system and carry warnings for increased risk of serious infections, death, and malignancies, the U.S. Food and Drug Administration added warnings for increased risk of blood clots and death associated with high-dose tofacitinib (10 mg twice daily), which is currently only approved for ulcerative colitis but was studied in patients treated for RA. ${ }^{44}$ A safety study of lower-dose tofacitinib (5 mg twice daily) is ongoing. Our findings regarding inpatient stays were diverse; fewer inpatient stays were observed for the injectable users relative to the oral users in the joint cohort alone.

Finally, there were no differences in ED visits between oral or injectable users across all condition cohorts. There is no previous evidence to show if medical utilization varies based on the route of administration in patients with chronic inflammatory conditions, although few studies have reported an increase in utilization when patients switch medications from their initially prescribed TNFi biologic. In a study of patients with RA, Vanderpoel et al. (2019) reported that patients who switched from their initial TNFi biologic to another TNFi or non-TNFi biologic had a higher tendency to encounter ED visits, and use physician visits and pharmacy services than patients who continued treatment. ${ }^{45}$ An earlier study by Baser et al. (2015) showed that when patients with RA switched from one TNFi to another TNFi biologic, the number of physician encounters in the 12-month follow-up were fewer compared with when patients switched from a TNFi to a non-TNFi biologic. ${ }^{46}$ However, from our study it is not known if the observed health care resource utilization differences at 
route of administration are clinically meaningful or a result of increased switching in the oral immunotherapy cohort.

While evidence supporting the advantage of injectable immunotherapies was observed, these injectable therapies were also found to be substantially more expensive, costing approximately $\$ 8,000-\$ 16,000$ more per year. Even in the joint conditions group, where relative effectiveness of injectable therapies was most pronounced, medical costs were still not enough to offset the higher cost of injectable therapy, thus, resulting in higher total costs. The relative differences in total costs were even higher for the skin conditions group and the joint and skin conditions group. Similar results were mirrored in studies comparing apremilast to biologics in patients with PsA and PSO. ${ }^{26,27}$ The average 12-month total health care cost for patients with PsA on apremilast was approximately $\$ 28,000$ versus $\$ 37,000$ for those on biologics and approximately $\$ 21,000$ versus $\$ 41,000$ for patients with PSO. ${ }^{26,27}$ Patients who respond well to oral medications may see sufficient treatment effect while incurring considerably lower costs.

\section{Limitations}

This study has several limitations, including that the study focused on injectable and oral medications but did not include infusible medications. An exploration of the claims database found more than $94 \%$ of patients would have indexed to infusible immunotherapies under the study design but were not naive to immunotherapy as of the index date. Hence, patients starting an infusible immunotherapy were not included, despite frequent use and importance as a therapeutic option in patients treated for joint, skin, or joint and skin conditions.

This was a real-world, retrospective analysis of claims data, not a prospective, randomized comparative study, so the influences of bias and confounding are of greater concern. Because this study analyzed administrative claims data, use of specific therapies may have been biased, in part, on real-world market conditions, physician prescribing practices, managed care formulary placement, and patient cost sharing. Propensity score methods were implemented to minimize potential selection bias arising from nonrandom treatment assignment. Even so, residual confounding due to unmeasured factors (e.g., disease severity) or imperfectly measured variables (e.g., persistence) may exist, so results should be interpreted with those limitations in mind.

\section{Conclusions}

This real-world comparative study found that patients initiating oral immunotherapy in the treatment of RA, PsA, and PSO were more likely to switch medications and have increased outpatient health care encounters relative to patients initiating injectable immunotherapies. Oral immunotherapy, however, was considerably less costly, which may be an essential consideration for treating patients who respond well to oral therapies.

\section{Authors}

RITUPARNA BHATTACHARYA, PhD; INSIYA POONAWALLA, PhD; SUVAPUN BUNNIRAN, PhD; and PHIL SCHWAB, PhD, Humana Healthcare Research, Humana, Louisville, Kentucky. KATIE HERREN, PharmD, MS, and ANDREA BLOOMFIELD, PharmD, BCPS, Humana, Louisville, Kentucky.

AUTHOR CORRESPONDENCE: Rituparna Bhattacharya, PhD, 515 W. Market St., Louisville, KY 40202. Tel.: 502.301.2163; Email: rbhattacharya@humana.com.

\section{DISCLOSURES}

This study was a Humana internal study, and all authors were at the time employees of Humana and used Humana resources. The authors have no conflicts of interest or financial interests to disclose that relate to the research described in this study.

This study was presented as a podium and poster presentation at the AMCP Managed Care \& Specialty Pharmacy Annual Meeting; April 23-26, 2018; Boston, MA.

\section{REFERENCES}

1. Hunter TM, Boytsov NN, Zhang X, Schroeder K, Michaud K, Araujo AB Prevalence of rheumatoid arthritis in the United States adult population in healthcare claims databases, 2004-2014. Rheumatol Int. 2017;37(9):1551-57.

2. Helmick CG, Lee-Han H, Hirsch SC, Baird TL, Bartlett CL. Prevalence of psoriasis among adults in the U.S.: 2003-2006 and 2009-2010 National Health and Nutrition Examination Surveys. Am J Prev Med. 2014;47(1):37-45.

3. Liu JT, Yeh HM, Liu SY, Chen KT. Psoriatic arthritis: epidemiology, diagnosis, and treatment. World J Orthop. 2014;5(4):537-43.

4. Schett G, Coates LC, Ash ZR, Finzel S, Conaghan PG. Structural damage in rheumatoid arthritis, psoriatic arthritis, and ankylosing spondylitis: traditional views, novel insights gained from TNF blockade, and concepts for the future. Arthritis Res Ther. 2011;13(Suppl 1):S4.

5. Lütze U, Archenholtz B. The impact of arthritis on daily life with the patient perspective in focus. Scand J Caring Sci. 2007;21(1):64-70.

6. Leino M, Mustonen A, Mattila K, Koulu L, Tuominen R. Influence of psoriasis on household chores and time spent on skin care at home: a questionnaire study. Dermatol Ther (Heidelb). 2015;5(2):107-16.

7. Singh JA, Saag KG, Bridges SL Jr, et al. 2015 American College of Rheumatology guideline for the treatment of rheumatoid arthritis. Arthritis Rheumatol. 2016;68(1):1-26.

8. Singh JA, Guyatt G, Ogdie A, et al. Special Article: 2018 American College of Rheumatology/National Psoriasis Foundation Guideline for the Treatment of Psoriatic Arthritis. Arthritis Rheumatol. 2019;71(1):5-32.

9. Elmets CA, Lim HW, Stoff B, et al. Joint American Academy of Dermatology-National Psoriasis Foundation guidelines of care for the management and treatment of psoriasis with phototherapy. J Am Acad Dermatol. 2019;81(3):775-804.

10. Menter A, Strober BE, Kaplan DH, et al. Joint AAD-NPF guidelines of care for the management and treatment of psoriasis with biologics. J Am Acad Dermatol. 2019;80(4):1029-72. 


\section{Comparing Medical Utilization and Cost Outcomes in Oral Versus Injectable Immunotherapy Users with Chronic Inflammatory Joint and Skin Diseases}

11. Walsh JA, Adejoro O, Chastek B, Palmer JB, Hur P. Treatment patterns among patients with psoriatic arthritis treated with a biologic in the United States: descriptive analyses from an administrative claims database. J Manag Care Spec Pharm. 2018;24(7):623-31. Available at: https://www.jmcp.org/ doi/10.18553/jmcp.2018.24.7.623

12. Harnett J, Wiederkehr D, Gerber R, Gruben D, Koenig A, Bourret J. Real-world evaluation of TNF-inhibitor utilization in rheumatoid arthritis. J Med Econ. 2016;19(2):91-102

13. Murage MJ, Anderson A, Casso D, et al. Treatment patterns, adherence, and persistence among psoriasis patients treated with biologics in a real-world setting, overall and by disease severity. J Dermatolog Treat. 2019;30(2):141-49.

14. Bolge SC, Goren A, Tandon N. Reasons for discontinuation of subcutaneous biologic therapy in the treatment of rheumatoid arthritis: a patient perspective. Patient Prefer Adherence. 2015;9:121-31.

15. Kavanaugh A, Helliwell P, Ritchlin CT. Psoriatic arthritis and burden of disease: patient perspectives from the population-based Multinational Assessment of Psoriasis and Psoriatic Arthritis (MAPP) Survey. Rheumatol Ther. 2016;3(1):91-102.

16. Lebwohl MG, Kavanaugh A, Armstrong AW, Van Voorhees AS. U.S perspectives in the management of psoriasis and psoriatic arthritis: patient and physician results from the population-based Multinational Assessment of Psoriasis and Psoriatic Arthritis (MAPP) Survey. Am J Clin Dermatol. 2016;17(1):87-97

17. Xeljanz (tofacitinib) tablets, for oral use. Pfizer. Revised May 2018. Available at: https://www.accessdata.fda.gov/drugsatfda_docs/ label/2018/203214s018lbl.pdf. Accessed September 8, 2020

18. Otezla (apremilast) tablets, for oral use. Celgene Corporation. Revised June 2017. Available at: https://www.accessdata.fda.gov/drugsatfda_docs/ label/2017/205437s006lbl.pdf. Accessed September 8, 2020

19. Fleischmann R, Mysler E, Hall S, et al. Efficacy and safety of tofacitinib monotherapy, tofacitinib with methotrexate, and adalimumab with methotrexate in patients with rheumatoid arthritis (ORAL Strategy): a phase 3b/4, double-blind, head-to-head, randomised controlled trial. Lancet. 2017:390(10093):457-68.

20. Kavanaugh A, Mease PJ, Gomez-Reino JJ, et al. Long-term (52-week) results of a phase III randomized, controlled trial of apremilast in patients with psoriatic arthritis. J Rheumatol. 2015;42(3):479-88.

21. Kavanaugh A, Mease PJ, Gomez-Reino JJ, et al. Treatment of psoriatic arthritis in a phase 3 randomised, placebo-controlled trial with apremilast, an oral phosphodiesterase 4 inhibitor. Ann Rheum Dis. 2014;73(6):1020-26.

22. Cutolo M, Myerson GE, Fleischmann RM, et al. A phase III, randomized, controlled trial of apremilast in patients with psoriatic arthritis: results of the PALACE 2 trial. J Rheumatol. 2016;43(9):1724-34

23. Edwards CJ, Blanco FJ, Crowley J, et al. Apremilast, an oral phosphodiesterase 4 inhibitor, in patients with psoriatic arthritis and current skin involvement: a phase III, randomised, controlled trial (PALACE 3). Ann Rheum Dis. 2016;75(6):1065-73.

24. Paul C, Cather J, Gooderham M, et al. Efficacy and safety of apremilast, an oral phosphodiesterase 4 inhibitor, in patients with moderate-to-severe plaque psoriasis over 52 weeks: a phase III, randomized controlled trial (ESTEEM 2). Br J Dermatol. 2015;173(6):1387-99.

25. Papp K, Reich K, Leonardi CL, et al. Apremilast, an oral phosphodiesterase 4 (PDE4) inhibitor, in patients with moderate to severe plaque psoriasis: results of a phase III, randomized, controlled trial (Efficacy and Safety Trial Evaluating the Effects of Apremilast in Psoriasis [ESTEEM] 1). J Am Acad Dermatol. 2015;73(1):37-49.
26. Wu JJ, Pelletier C, Ung B, Tian M. Treatment patterns and healthcare costs among biologic-naive patients initiating apremilast or biologics for the treatment of psoriatic arthritis: results from a U.S. claims analysis. Curr Med Res Opin. 2020;36(1):169-176.

27. Wu JJ, Pelletier C, Ung B, Tian M. Real-world treatment patterns and healthcare costs among biologic-naive patients initiating apremilast or biologics for the treatment of psoriasis. J Med Econ. 2019;22(4):365-71.

28. Machado MAÁ, Moura CS, Guerra SF, Curtis JR, Abrahamowicz M, Bernatsky S. Effectiveness and safety of tofacitinib in rheumatoid arthritis: a cohort study. Arthritis Res Ther. 2018;20(1):60.

29. Harnett J, Gerber R, Gruben D, Koenig AS, Chen C. Evaluation of realworld experience with tofacitinib compared with adalimumab, etanercept, and abatacept in RA patients with 1 previous biologic DMARD: data from a U.S. administrative claims database. J Manag Care Spec Pharm. 2016;22(12):1457-71. Available at: https://www.jmcp.org/doi/10.18553/ jmcp.2016.22.12.1457.

30. U.S. Census Bureau. Census regions and divisions of the United States. Cited March 29, 2017. Available at: http://www2.census.gov/geo/pdfs/mapsdata/maps/reference/us_regdiv.pdf. Accessed September 8, 2020.

31. Hart LG, Larson EH, Lishner DM. Rural definitions for health policy and research. Am J Public Health. 2005;95(7):1149-55.

32. West AN, Weeks WB, Wallace AE. Rural veterans and access to highquality care for high-risk surgeries. Health Serv Res. 2008;43(5 Pt 1):1737-51.

33. Deyo RA, Cherkin DC, Ciol MA. Adapting a clinical comorbidity index for use with ICD-9-CM administrative databases. J Clin Epidemiol. 1992;45(6):613-19.

34. Quan H, Sundararajan V, Halfon P, et al. Coding algorithms for defining comorbidities in ICD-9-CM and ICD-10 administrative data. Med Care. 2005;43(11):1130-39.

35. Klabunde CN, Potosky AL, Legler JM, Warren JL. Development of a comorbidity index using physician claims data. J Clin Epidemiol. 2000;53(12):1258-67.

36. Sloan KL, Sales AE, Liu CF, et al. Construction and characteristics of the RxRisk-V: a VA-adapted pharmacy-based case-mix instrument. Med Care. 2003;41(6):761-74

37. Deyo RA, Cherkin DC, Ciol MA. Adapting a clinical comorbidity index for use with ICD-9-CM administrative databases. J Clin Epidemiol. 1992;45(6):613-19.

38. Centers for Medicare \& Medicaid Services. Provider taxonomy. March 29, 2017. Available at: https://www.cms.gov/Medicare/Provider-Enrollmentand-Certification/MedicareProviderSupEnroll/Taxonomy. Accessed September 8, 2020.

39. Krack G, Zeidler H, Zeidler J. Claims data analysis of tumor necrosis factor inhibitor treatment dosing among patients with rheumatoid arthritis: a systematic review of methods. Drugs Real World Outcomes. 2016;3(3):265-78

40. Fisher MD, Watson C, Fox KM, Chen YW, Gandra SR. Dosing patterns of three tumor necrosis factor blockers among patients with rheumatoid arthritis in a large United States managed care population. Curr Med Res Opin. 2013;29(5):561-68.

41. Wu E, Chen L, Birnbaum H, Yang E, Cifaldi M. Retrospective claims data analysis of dosage adjustment patterns of TNF antagonists among patients with rheumatoid arthritis. Curr Med Res Opin. 2008;24(8):2229-40.

42. Austin PC. An introduction to propensity score methods for reducing the effects of confounding in observational studies. Multivariate Behav Res. 2011;46(3):399-424 
43. Curtis JR, Baddley JW, Yang S, et al. Derivation and preliminary validation of an administrative claims-based algorithm for the effectiveness of medications for rheumatoid arthritis. Arthritis Res Ther. 2011;13(5):R155.

44. U.S. Food and Drug Administration. FDA approves Boxed Warning about increased risk of blood clots and death with higher dose of arthritis and ulcerative colitis medicine tofacitinib (Xeljanz, Xelijanz XR). July 26, 2019. Available at: https://www.fda.gov/drugs/drug-safety-and-availability/ fda-approves-boxed-warning-about-increased-risk-blood-clots-and-deathhigher-dose-arthritis-and. Accessed September 8, 2020.
45. Vanderpoel J, Tkacz J, Brady BL, Ellis L. Health care resource utilization and costs associated with switching biologics in rheumatoid arthritis. Clin Ther. 2019;41(6):1080-89.e5.

46. Baser O, Ganguli A, Roy S, Xie L, Cifaldi M. Impact of switching from an initial tumor necrosis factor inhibitor on health care resource utilization and costs among patients with rheumatoid arthritis. Clin Ther. 2015;37(7):1454-65.

\section{Correction}

Effectiveness and Costs Among Rheumatoid Arthritis Patients Treated with Targeted Immunomodulators Using Real-World U.S. Data.

Gharaibeh M, Bonafede M, McMorrow D, Hernandez EJM, Stolshek BS. J Manag Care Spec Pharm. 2020;26(8):1039-49.

The authors would like to make the following corrections to the above article:

Page 1042, the second paragraph, under Nonadherence, should be corrected as shown (change shown in bold):

"For self-administered drugs (abatacept administered subcutaneously [SC], adalimumab, certolizumab pegol, etanercept, golimumab SC, and tocilizumab SC) and tofacitinib, nonadherence was defined as medication possession ratio (MPR) of $\leq 80 \%$. MPR was calculated as the total days supply for the index therapy divided by the total follow-up days (365 days fixed follow-up period). If a patient refilled a medication before the previous days supply running out, the days supply was extended for a maximum of 14 days (allowing for 14 days of overlap). For infused drugs (abatacept, golimumab, and tocilizumab), a proportion of days covered (PDC) of $\leq 80 \%$ was calculated using the expected duration of clinical benefit."

While the authors regret these errors, they do not affect the conclusions of the article. 